\title{
Analysis of background parenchymal enhancement (BPE) on contrast enhanced spectral mammography compared with magnetic resonance imaging
}

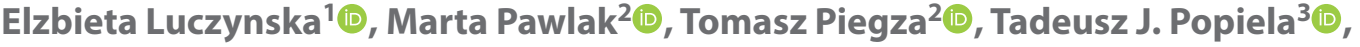 \\ Sylwia Heinze2 ${ }^{10}$, Sonia Dyczek ${ }^{4}$, Wojciech Rudnicki ${ }^{5}$ \\ ${ }^{1}$ University of Rzeszow, Faculty of Medicine, Rzeszow, Poland \\ ${ }^{2}$ Maria Sklodowska-Curie National Research Institute of Oncology in Cracow, Poland \\ ${ }^{3}$ Chair of Radiology, Jagiellonian University Medical College, Cracow, Poland \\ ${ }^{4}$ University Hospital in Cracow, Poland \\ ${ }^{5}$ Jagiellonian University Medical College, Cracow, Poland
}

\begin{abstract}
Objectives: With the growing number of new breast cancer cases in women, new methods of imaging arise. Contrast enhanced spectral mammography (CESM) and magnetic resonance imaging (MRI) are comparable methods regarding sensitivity. The aim of this study is to check if analysis of background parenchymal enhancement on CESM can improve its usefulness.

Material and methods: A total of 64 patients with breast lesions found previously on ultrasound or mammography underwent MRI and CESM within less than one month. On MRI the contrast enhancement kinetics and visual BPE were evaluated. On CESM the enhancement of lesions was noted as well as a quantitative level of BPH. The gathered data was analysed in terms of patterns and relations.

Results: A total of 66 lesions were identified both on MRI and CESM, including 11 (17\%) benign and 55 (83\%) malignant lesions. Among malignant lesions $13(20 \%)$ were assessed as intraductal and $42(64 \%)$ as infiltrating carcinomas. The study showed correlation between the level of enhancement on CESM and the type of kinetic curve on MRI and lesion enhancement on CESM as well as confirmed the fact that the BPE is a destimulant in both methods of imaging.

Conclusions: Evaluation of BPE level on CESM can help reading radiologists to define a lesion as malignant with higher probability than based only on the qualitative lesion enhancement level.
\end{abstract}

Key words: breast cancer; mri; cesm; diagnostic imaging; breast; cancer; mammography

Ginekologia Polska 2021; 92, 2: 92-97

\section{INTRODUCTION}

Nowadays, breast cancer is the most frequent type of cancer in women in the European Union countries (29.2\% of all cancers) [1]. Early detection of cancer significantly increases the chance for cure [2]. Therefore, at the time of fast developing technology, basic imaging methods such as ultrasonography and mammography were complemented by MRI (magnetic resonance imaging) and more recently by CESM (contrast enhanced spectral mammography), accepted by FDA in 2011.

Both methods are stated to have comparable sensitivity and specificity described in numerous studies, which enables more diagnostic possibilities in a bigger number of patients. Above examinations can be applied interchange- ably in cases when the patients report contraindications to any of them [3-5]. This fact emphasises the need of exploring all the aspects of every new method, including CESM.

Breast parenchymal enhancement (BPE) is a feature routinely evaluated on MRI [6]. MRI reports include one of four BPE values measured qualitatively: minimal, mild, moderate, marked and symmetry of enhancement in both breasts. A correlation between BPE on MRI and breast cancer risk was found. The stronger the enhancement, the higher the risk [7]. What is more, lesion evaluation on the background of strongly marked BPE is much more difficult for a radiologist describing the findings.

Similarity of this imaging method to CESM leads to the assumption that this parameter should be also measurable 


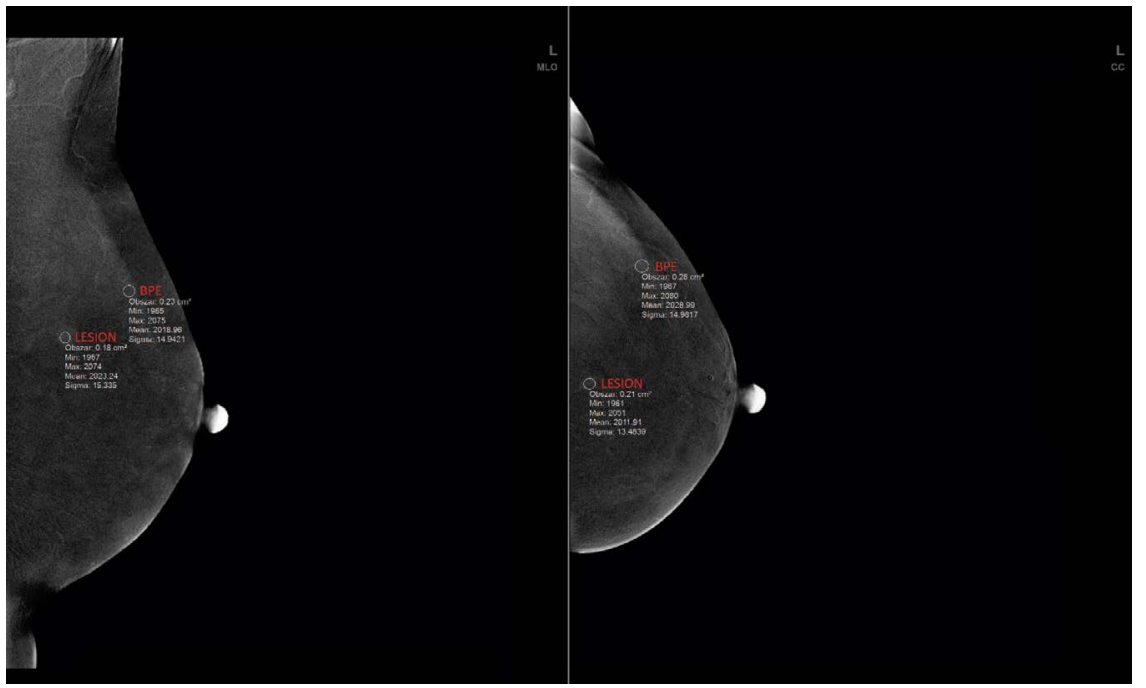

Figure 1. Measurement of BPE value and lesion enhancement using an ellipse shaped Region of Interest (ROI) on contrast enhanced spectral mammography (CESM) from Figure 2

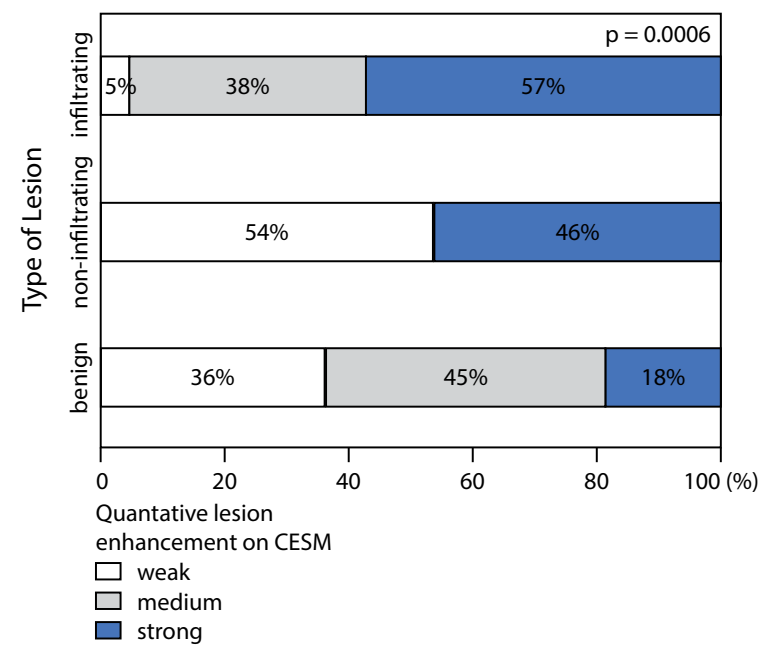

Figure 2. Relation between qualitative lesion enhancement on contrast enhanced spectral mammography (CESM) and histopathological result

on CESM. So far, the studies analysed BPE only in terms of finding if its value can determine the risk of breast cancer $[8,9]$. The outcomes of these analyses indicate that the parameter should be taken into consideration in the final assessment of examination. Up to now BPE on CESM has not been compared to BPE on MRI in the same patients. The aim of our study is to determine the usefulness of BPE evaluation on CESM. We also checked if it allows radiologists to assess lesions and exclude the probability of neoplastic process with greater certainty.

This study was performed in compliance with the Declaration of Helsinki and it received the approval of the Ethical Committee at the Regional Medical Chamber (acceptance No. OIL/KBL/17/2018).

\section{MATERIAL AND METHODS}

Both examinations, CESM and MRI were performed on 84 patients in 2018. Patients suspicious of multifocality and multicentricity or in case of other diagnostic doubts such as heterogeneous or dense breast anatomy underwent CESM examination. MRI examination followed CESM if the diagnostic problem was still present or if histopathologic verification was necessary for lesions visible only after contrast administration. Within the observed group the enhancement visible on CESM was not confirmed on MRI in 5 cases. Another 6 patients were referred to chemotherapy due to extension of the disease process and receptor status and 7 patients underwent surgical procedure in another Oncology Clinic. As a result, the analysis comprises 64 patients who underwent breast CESM and MRI.

For CESM a protocol routinely used in the department was applied with GE Senographe Essential machine. Contrast medium administration was performed with an automatic syringe Optivantage DH. Dose of contrast media (iopromide a $370 \mathrm{mg} / \mathrm{mL}$ ) was calculated following the formula $1.5 \mathrm{~mL} / \mathrm{kg}$ of body weight. Patients without any known contradictions were given a bolus of contrast with a rate of $3 \mathrm{~mL} / \mathrm{s}$ followed by a chaser of $\mathrm{NaCl} 0.9 \%$ solution. At that moment, a timer was started and after 2 minutes the first image acquisition was performed. A total of 4 exposures were performed in the following order: craniocaudal (CC) of the breast with smaller probability of cancer based on preliminary diagnostic imaging, $\mathrm{CC}$ of the more suspicious breast, mediolateral oblique (MLO) of first breast and then MLO of the second breast.

Lesion enhancement was evaluated on subtraction images and described qualitatively as weak, medium or strong as well as qualitatively using the Region of Interest (ROI) tool. 
$\mathrm{ROI}$ is a part of the used Senolris software and gives information of the minimum, maximum and average pixel brightness distribution within the shape of our choosing (oval was used in this study). Since there are only 2 time-points of contrast kinetics measured, there is no possibility to assess a kinetic curve like in breast MRI.

BPE on CESM was measured with the ROI with an oval shape in the most homogenous part of parenchyma both in CC and MLO views of the breast with the suspicious lesion (Fig. 1). Afterwards, the values were divided into 4 equal ranges and described as minimal, mild, moderate and marked to adapt the scale to the one used in MRI (ACR BI-RADS standards for reporting).

In order to compare BPE on CESM and MRI qualitative assessment was performed based on enhancement values measured with ROI. For every patient mean BPE was calculated for CC and MLO views. The obtained results ranged from 2000.7 to 2067.1. As a next step, the enhancement values were divided into four scopes, following the BI-RADS standards for BPE evaluation on MRI: minimal ranging from 2000.7-2017.3, mild - 2017.4-2033.9, moderate - 2034.0-2050.5, marked - 2050.6-2067.1.

MRI examination was performed using Siemens Avanto 1,5T machine. Patients without any known contradictions were qualified for the examination. There was a strict time requirement of menstrual cycle for the patients to be scheduled for the examination - the day of the procedure was within 5-12 day of the cycle. If the patient underwent hysterectomy, they had their progesterone level evaluated before the exam. Only patients with the progesterone level within follicular phase of the menstrual cycle were qualified for the study. The protocol used included T1WI,T2WI — with and without Fat Suppression (FATSAT), Diffusion Weighted Images (DWI), and dynamic T1-weighted 3D sequence after contrast media administration. Contrast medium (gadobutrolum a $0.60472 \mathrm{~g} / \mathrm{mL}$ ) was injected using Optistar Elite automatic syringe. The amount of contrast was calculated according to $0.1 \mathrm{~mL} / \mathrm{kg}$ of body weight formula.

Lesion kinetic curve assessment was performed afterwards using Siemens syngo ${ }^{\circledR}$ software and described as persistent, plateau or wash-out.

\section{Histopathological examination}

All lesions were histopathologically verified. Following biopsy, methods were used to obtain lesion samples: core-needle biopsy, vacuum assisted breast biopsy, stereotactic biopsy guided on MG/MRI or lumpectomy. Standard hematoxylin and eosin staining was followed by histopathological examination by pathologists experienced in breast diseases.

Histopathological examination confirmed the presence of one focal lesion in all 66 patients on both CESM and MRI. Among all diagnosed lesions 55 (83\%) were malignant in- cluding 42 (64\%) infiltrating lesions, and 13 (20\%) non-infiltrating lesions. The remaining 11 (17\%) lesions were benign.

\section{Statistical methods}

Statistica software and following statistical methods were used for calculations:

- chi-squared dependence test to compare lesion enhancement on CESM and its histopathological character; BPE parameter assessment both on CESM and MRI in terms of its histopathological type; assess the influence of BPE on MRI on enhancement curve type on MRI

- ROC analysis to check if quantitative lesion enhancement on CESM and lesion enhancement curve type on MRI depends on histologically determined cancer stage

- Kruskal-Wallis test ANOVA to assess the relation between ratio of quantitative BPE value on CESM to quantitative lesion CESM enhancement and qualitative lesion enhancement on CESM; assess the relation between qualitative BPE on CESM and BPE on MRI; compare the quantitative BP enhancement indicator on CESM and quantitative lesion enhancement on CESM to BPE on MRI

- Mann-Whitney U test to compare the quantitative BP enhancement indicator on CESM and quantitative lesion enhancement on CESM to lesion enhancement curve on MRI

\section{RESULTS}

\section{CESM qualitative enhancement of the lesion}

Based on CESM results, weak enhancement was determined for 12 (18\%) lesions, medium for 21 (32\%), and strong for 33 (50\%). Strong enhancement was more frequent among malignant lesions than benign ( $56 \%$ vs $18 \%$ ). This relation was statistically significant (proportion test $\mathrm{p}=0.02$ ). However, the number of benign cases classified with strong enhancement was only two. The relation between lesion type (malignant and benign) and enhancement level was not statistically significant (chi-squared dependence test $p=0.054)$.

Lesion enhancement level depends on lesion type only if the classification of the lesions is extremely specific, i.e. infiltrating and non-infiltrating benign lesions (chi-squared dependence test $p=0.0006$ ). The higher the level of malignancy, the more often the enhancement visible on CESM was described as strong. Consequently, the more benign the lesion was, the more often the enhancement was described as weak on CESM. This is shown in Figure 2.

There was also a significant difference between median value of quantitative lesion enhancement on CESM and histopathological result ( $U$ Manna-Whitney test $p=0.03$ ) and lesion type (Kruskal-Wallis test $p=0.05$ ). Figure 3 shows that there has been a gradual increase in the median value of lesion enhancement on CESM with rising the degree of 


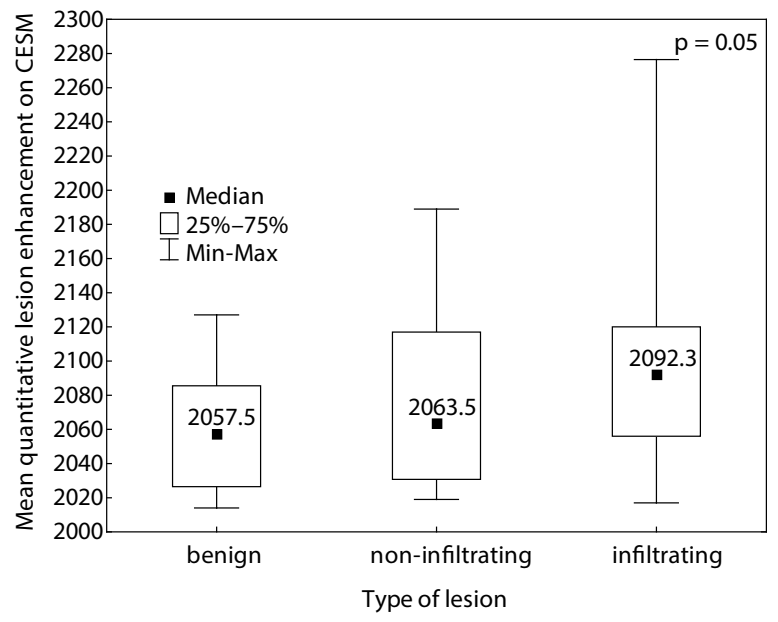

Figure 3. Relation between qualitative lesion enhancement on contrast enhanced spectral mammography (CESM) and histopathological result

malignancy of the lesion. Therefore, the result for comparison of qualitative and quantitative lesion enhancement on CESM with lesion type is comparable.

As a next step quantitative BPE value on CESM was compared. In the analysed material, median value of BPE on CESM was 2030.11 - minimum 2000.7, maximum 2067.1. BPE value was neither related to the type of lesion nor to its malignancy as shown in Figure 4A and 4B.

The enhancement values on CESM were analysed with ROC to determine whether the lesions could be described as malignant on their basis and what range of values would be adequate. Based on quantitative and qualitative enhancement it was possible to determine the lesion's malignancy
Table 1. AUC, $p$ and threshold values for calculation of lesion enhancement and BPE

\begin{tabular}{|l|l|l|l|}
\hline Parameter on CESM & AUC & $\mathbf{p}$ & Treshold value \\
\hline $\begin{array}{l}\text { Qualitative lesion } \\
\text { enhancement }\end{array}$ & 0.711 & 0.0110 & Strong \\
\hline $\begin{array}{l}\text { Quantitive lesion } \\
\text { enhancement }\end{array}$ & 0.712 & 0.0077 & 2091.5 \\
\hline BPE quantitive* & 0.582 & 0.3398 & 2018.86 \\
\hline BPE qualitive* & 0.569 & 0.4191 & minimum \\
\hline Ratio/indicato* & 0.746 & 0.0004 & 97.3 \\
\hline
\end{tabular}

* destimulant; AUC — area under curve; CESM — contrast enhanced spectral mammography; BPE - background parenchymal enhancement

$(p<0.01$ AUC). The malignancy can be determined based on percentage ratio of BPE value on CESM to lesion CESM enhancement $(p=0.0004)$. The described ratio/indicator is incredibly useful in differentiating between benign and malignant lesions - AUC [(area under curve) is the biggest, $p$ is the lowest and the estimated sensitivity, specificity and accuracy based on threshold value are the highest. This ratio/indicator is a distimulant, which means that the lower its value, the higher the malignancy of the lesion. The results of analysis showed that for values smaller or equal $97.3 \%$ lesions can be described as malignant. All values are shown in Table 1.

The results show that the variable is a destimulant, which means that the lower its value, the higher the malignancy of the lesion. Further step involved determining whether quantitative lesion enhancement value on CESM depends on qualitative BPE on CESM. This relation is presented in Figure 5.
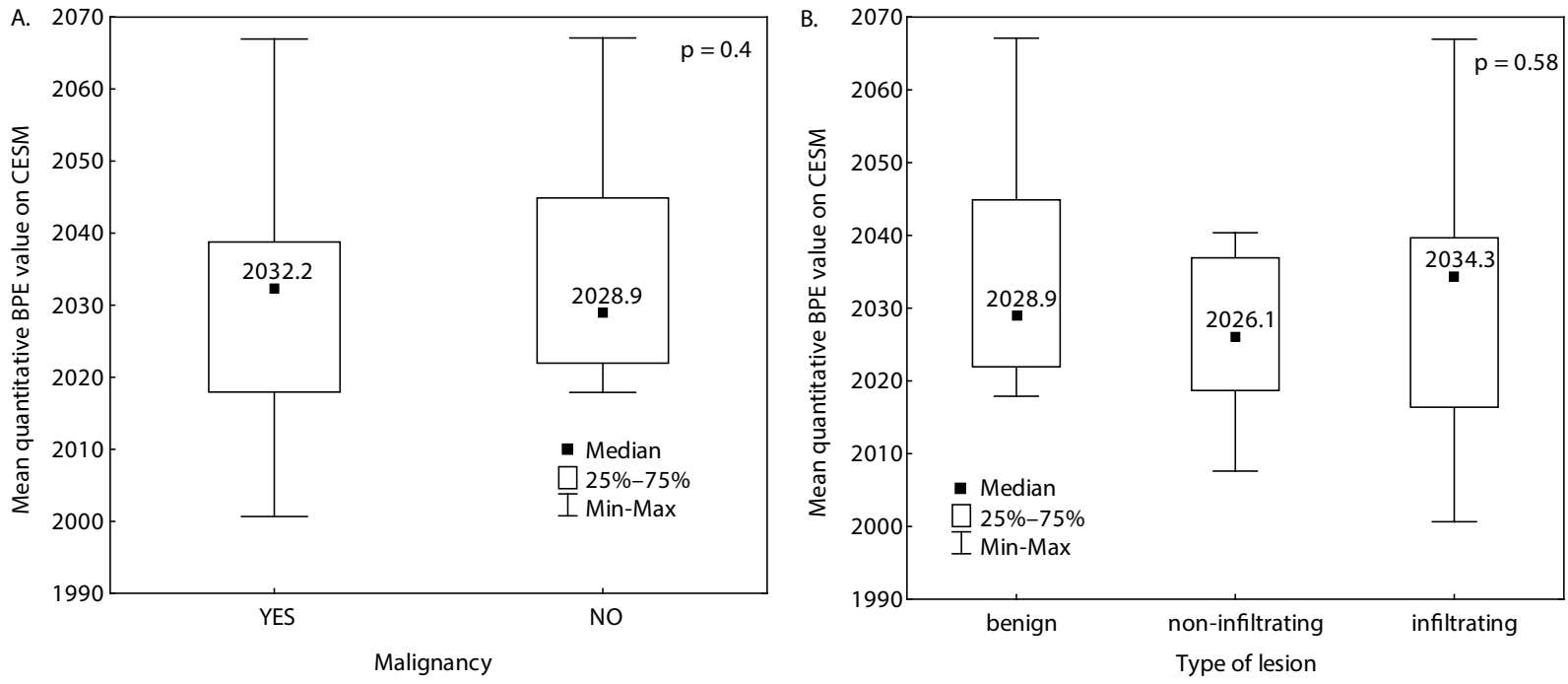

Figure 4. Relation between mean quantitative background parenchymal enhancement (BPE) value on contrast enhanced spectral mammography (CESM) and malignancy (A) and type of lesion (B) 


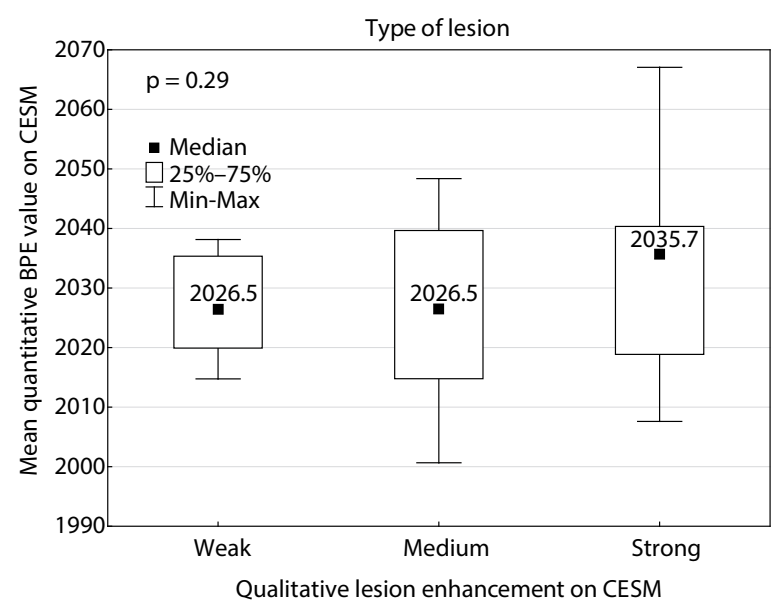

Figure 5. Relation between mean quantitative background parenchymal enhancement (BPE) value on contrast enhanced spectral mammography (CESM) and qualitative lesion enhancement on CESM

There is a strong correlation between BPE qualitative enhancement on CESM and lesion qualitative enhancement on CESM ratio and qualitative enhancement (Kruskal Wallis test ANOVA $p<0.001)$. The smaller the share - the ratio value - the stronger enhancement on CESM. Additionally, the rank correlation between mean enhancement on CESM and mean BPE enhancement was evaluated $(p=0.01)$. Correlation coefficient was 0.31 .

\section{MRI qualitative BPE and lesion enhancement}

BPE was determined as minimum on MRI in 16 (24\%) cases, mild in 33 (50\%) cases, moderate in 13 (19\%) cases and marked in $4(6 \%)$ cases. Both BPE on CESM and BPE on MRI do not reveal malignancy of the detected lesion neither imply the particular type of lesion (chi-squared dependence test $p=0.93$ and 0.99 respectively). Taking $p$ into account, enhancement and lesion type are not related to parenchymal enhancement.

It was analysed to which extent curve type on MRI and level of BP enhancement could be applied to determine lesion malignancy. Based on the curve type on MRI it is possible to decide if the lesion is malignant (ROC analysis $\mathrm{p}<0.001$ ), i.e. reference level type III (wash-out). Parenchymal enhancement level on MRI, similarly to BPE on CESM does not allow to determine malignancy $(p=0.69)$. This variable is also destimulant. This is shown in Table 2 .

Material analysis revealed a lack of correlation between curve type on MRI and BPE on MRI (chi-squared dependence test $p=0.68$ ). Type II of kinetic curve in lesion was seen in patients with PBE on MR described as: minimal in 31\%, mild in $50 \%$, moderate in $15 \%$, marked in $4 \%$ of total cases. Type III was seen in lesion with BPE described as: minimal in $20 \%$, mild in $50 \%$ moderate in $23 \%$, marked in $7 \%$ of cases.

It was also proved that mean qualitative BPE on CESM is not related to BPE level on MRI (Kruskal Wallis test ANOVA $p=0.06)$. Figure 6 shows the values.
Table 2. AUC parameter, $p$ and threshold value for calculations of MRI kinetic curve and BPE in MRI

\begin{tabular}{|l|l|l|l|}
\hline Parameter & AUC & $\mathbf{p}$ & Treshold value \\
\hline $\begin{array}{l}\text { Qualitative } \\
\text { enhancement }\end{array}$ & 0.864 & 0.0000 & Type III \\
\hline $\begin{array}{l}\text { Quantitative } \\
\text { enhancement }\end{array}$ & 0.538 & 0.6861 & moderate \\
\hline
\end{tabular}

AUC - area under curve

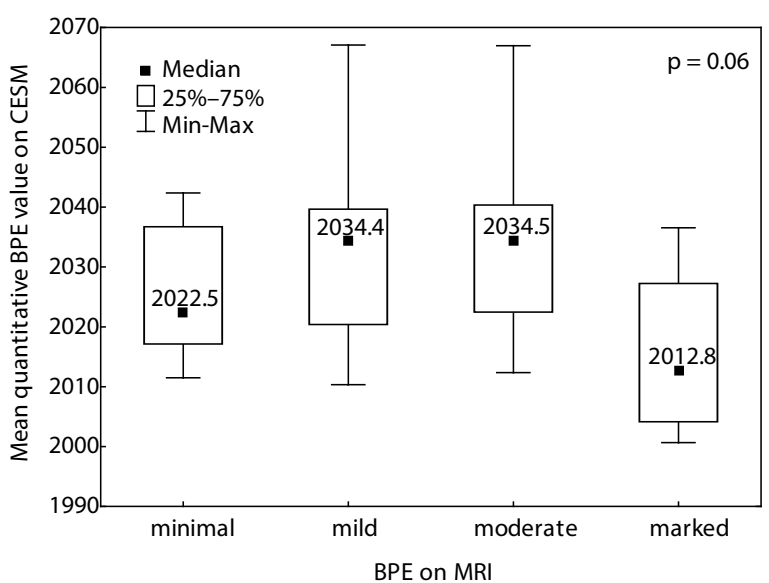

Figure 6. Relation between mean quantitative background parenchymal enhancement (BPE) value on contrast enhanced spectral mammography (CESM) and BPE on magnetic resonance imaging (MRI)

Comparison of BPE on CESM to mean enhancement ratio with the results of $M R I$ examination shows that the ratio depends on curve type on MRI (U Mann-Whitney test $p=0.0004)$. The ratio is lower for lesions with determined curve type III on MRI but is not related to BPE level on MRI (Kruskal-Wallis test ANOVA $p=0.63$ ). Figure $7 a$ and $7 \mathrm{~b}$ show the relations.

The analysis also revealed a lack of relation between qualitative BPE on MRI and CESM. The assessments of both methods were concordant in only 20 cases (30\%) - i.e. that many cases were evaluated the same on both methods of examination. It is worth noting, that none of the cases determined as marked on CESM was determined as marked on MRI. The cases described as marked on MRI were characterised by the lowest BPE value.

\section{DISCUSSION}

The study revealed that analysis of BPE on CESM brings tangible results. It appeared that this feature is a destimulant both on MRI and CESM. It can be said that it creates noise which deteriorates the visibility of lesions in breast imaging examinations. Therefore, the assessment of this parameter allows reading radiologists to determine breast cancer probability with greater accuracy. 

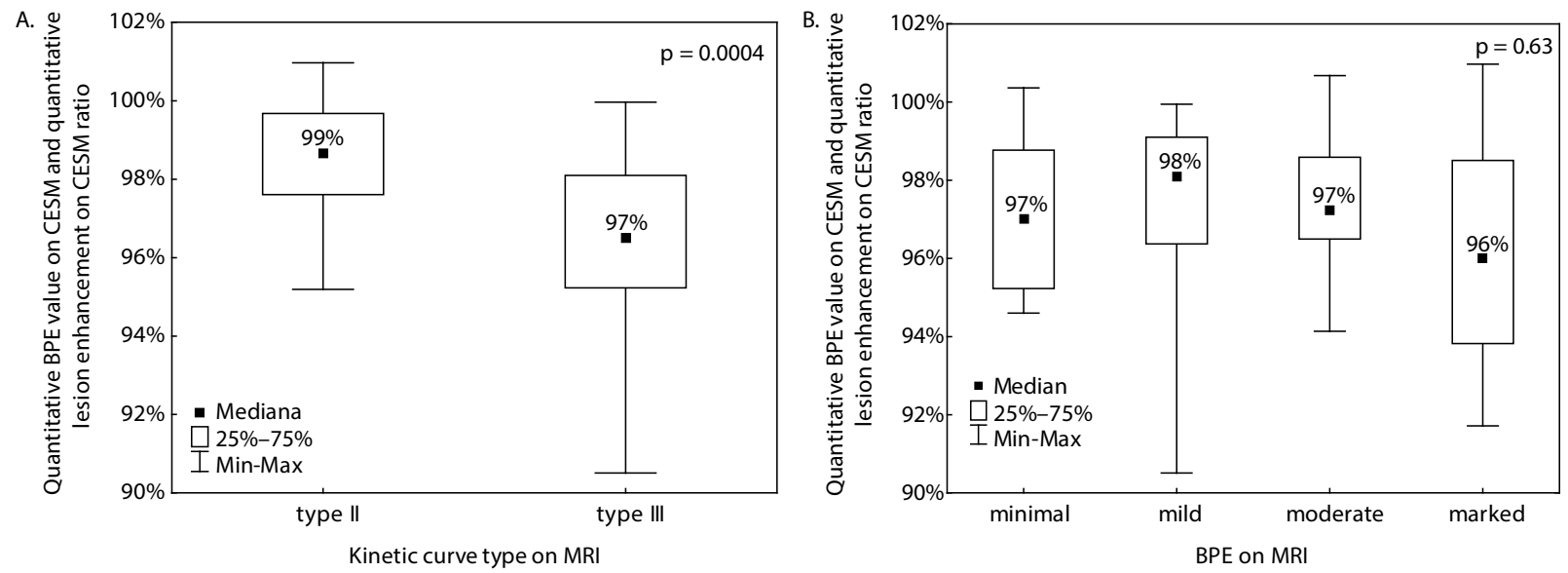

Figure 7. Relation between quantitative background parenchymal enhancement (BPE) value on contrast enhanced spectral mammography (CESM) and quantitative lesion enhancement on CESM ratio and lesion enhancement curve type II and III on magnetic resonance imaging (MRI) (A) BPE on MRI (B)

Our findings are not consistent with the results of previous study [10], where BPE did not affect lesion detection efficacy on CESM. It is important to emphasise that in our study quantitative BP and lesions enhancement were evaluated for the first time, which could have influenced the discrepancy between the results. This issue requires further analysis.

Since mammography examination is two-dimensional, BPE is not reproduced on CESM in the same way as on MRI. That is the reason why quantitative assessment brings advantages and increases repeatability.

As a limitation of our study we should mention a lack of data on the menstruation cycle day of the patients admitted for CESM and carefully selected date of MRI examination. However, it results from the studies $[3,11]$ that the menstrual cycle does not influence BPE on CESM significantly and it does not fluctuate during the cycle. Another limitation is a small group of analysed patients. It stems from a retrospective form of conducted analysis and limited number of patients who underwent both examinations due to clinical indications before treatment.

\section{CONCLUSIONS}

Our study suggests that evaluation of BPE level on CESM can help radiologists to define a lesion as malignant with higher probability than based only on the qualitative lesion enhancement level and should be evaluated for each patient.

\section{Conflicts of interest}

The Authors declare that no conflicts of interest exist in terms of publication of this article.

\section{Authors' contributions}

E.L. developed and researched the concept, collected and performed the data analysis and edited the text. T.P and M.P. collected the data. S.H., T.J.P., S.D. contributed to the final version of the manuscript. W.R. edited text and supervised the study.

\section{REFERENCES}

1. Dafni U, Tsourti Z, Alatsathianos I. Breast Cancer Statistics in the European Union: Incidence and Survival across European Countries. Breast Care (Basel). 2019; 14(6): 344-353, doi: 10.1159/000503219, indexed in Pubmed: 31933579.

2. Kalager $M$, Haldorsen $T$, Bretthauer $M$, et al. Improved breast cancer survival following introduction of an organized mammography screening program among both screened and unscreened women: a population-based cohort study. Breast Cancer Res. 2009; 11(4): R44, doi: 10.1186/bcr2331, indexed in Pubmed: 19575807.

3. Fallenberg EM, Schmitzberger FF, Amer H, et al. Contrast-enhanced spectral mammography vs. mammography and MRI - clinical performance in a multi-reader evaluation. Eur Radiol. 2017; 27(7): 2752-2764, doi: 10.1007/s00330-016-4650-6, indexed in Pubmed: 27896471.

4. Covington MF, Pizzitola VJ, Lorans R, et al. The Future of Contrast-Enhanced Mammography. AJR Am J Roentgenol. 2018; 210(2): 292-300, doi: 10.2214/AJR.17.18749, indexed in Pubmed: 29064748.

5. Barra FR, Sobrinho AB, Barra RR, et al. Contrast-Enhanced Mammography (CEM) for Detecting Residual Disease after Neoadjuvant Chemotherapy: A Comparison with Breast Magnetic Resonance Imaging (MRI). Biomed Res Int. 2018; 2018: 8531916, doi: 10.1155/2018/8531916, indexed in Pubmed: 30533440 .

6. Morris EA, Comstock CE, Lee $\mathrm{CH}$. et al.. ACR BI-RADS ${ }^{\oplus}$ Magnetic Resonance Imaging. In: ACR BI-RADS ${ }^{\circledR}$ Atlas, Breast Imaging Reporting and Data System. American College of Radiology, Reston, VA 2013.

7. King V, Brooks JD, Bernstein JL, et al. Background parenchymal enhancement at breast MR imaging and breast cancer risk. Radiology. 2011; 260(1): 50-60, doi: 10.1148/radiol.11102156, indexed in Pubmed: 21493794.

8. Sorin V, Yagil Y, Shalmon A, et al. Background Parenchymal Enhancement at Contrast-Enhanced Spectral Mammography (CESM) as a Breast Cancer Risk Factor. Acad Radiol. 2020; 27(9): 1234-1240, doi: 10.1016/j. acra.2019.10.034, indexed in Pubmed: 31812577.

9. Savaridas SL, Taylor DB, Gunawardana D, et al. Could parenchymal enhancement on contrast-enhanced spectral mammography (CESM) represent a new breast cancer risk factor? Correlation with known radiology risk factors. Clin Radiol. 2017; 72(12): 1085.e1-1085.e9, doi: 10.1016/j.crad.2017.07.017, indexed in Pubmed: 28870431.

10. Kim G, Phillips J, Cole E, et al. Comparison of Contrast-Enhanced Mammography With Conventional Digital Mammography in Breast Cancer Screening: A Pilot Study. J Am Coll Radiol. 2019; 16(10): 1456-1463, doi: 10.1016/j.jacr.2019.04.007, indexed in Pubmed: 31092346.

11. Zhao $S$, Zhang $X$, Zhong $H$, et al. Background Parenchymal Enhancement on Contrast-Enhanced Spectral Mammography: Influence of Age, Breast Density, Menstruation Status, and Menstrual Cycle Timing. Sci Rep. 2020; 10(1): 8608, doi: 10.1038/s41598-020-65526-8, indexed in Pubmed: 32451404. 